\title{
An Influence of Geometrical Parameters of the Lock of the Blade-Disc Joint on Stress in the FEA and DIC Methods
}

\author{
Adam Kozakiewicz', Olga Grzejszczak ${ }^{1 *}$ \\ 1 Faculty of Mechatronics, Armament and Aerospace, Military University of Technology, gen. Sylwestra \\ Kaliskiego 2, 00-908 Warszawa, Poland \\ ${ }^{*}$ Corresponding author's email: olga.grzejszczak@wat.edu.pl
}

\begin{abstract}
In this paper, an assessment of strength properties of the existing blade-disc dovetail joint was presented, taking the parametric optimization of its geometry. A sensitivity of the analysis was carried out to determine the relationship between the selected geometric parameters and strength properties of the structure questioned. The results from experiments and computer simulations were compared. In addition, the fatigue life of the structure was analyzed for different materials and using the change of speed ranges and parameterization of geometry.
\end{abstract}

Keywords: parameterization, strength analysis, dovetail joint, DIC, FEA, stress distribution.

\section{INTRODUCTION}

A rotor assembly is a basic component of a turbine jet engine. Usually, this element, including a compressor, is subjected to centrifugal force [16]. The value of this loading results from the operating conditions, i.e. it strongly depends on the rotational speed of the rotor. The transferred loading depends on a temperature during exploitation, kind of material as well as geometry of the structural system. The numerical approach to the lock region containing partial parameterization of geometry by means the finite element method (FEM) can be found in a lot of papers $[1,17,22]$. In the case of lock connections used in compressor rotors, the correctly selected geometrical features of the zone allow reducing the stress [12]. From the operation point of view, the resistance on highcycle fatigue plays an important role. Therefore, determination of the fatigue durability is considered in many research groups $[4,7,10,11,12$, $15,19,23,29]$. These authors analyze the fatigue durability of rotor elements at various operational stages containing additional working conditions: (Fig. 1), corrosion, defects caused by collisions with sucked-in foreign bodies (scratches, cracks), abrasive effects (so-called fretting) up to failure. Therefore, the aim of the paper focuses on the assessment of fatigue compressor rotor using experimental and numerical approaches.

The assessment of strength properties of the blade-disc dovetail joint was carried out, taking into account the parametric optimization of its geometry. The analysis was performed to examine the relationship between the selected geometric features and the strength properties of the structure. It was done by comparing the results from experiments and simulations. In addition, the fatigue durability of the structure was analyzed selecting different materials and speed ranges, as well as parameterization of the geometry.

\section{FE model}

In the finite element method, or analysis, (FEM or FEA) the discretization of region is an essential step to help the finite element method solve the governing differential equations by approximating the solution within each finite element [24]. Mesh generation, as a preprocessing step, involves discretization of a solid model into a set of points called nodes on which the numerical solution is 


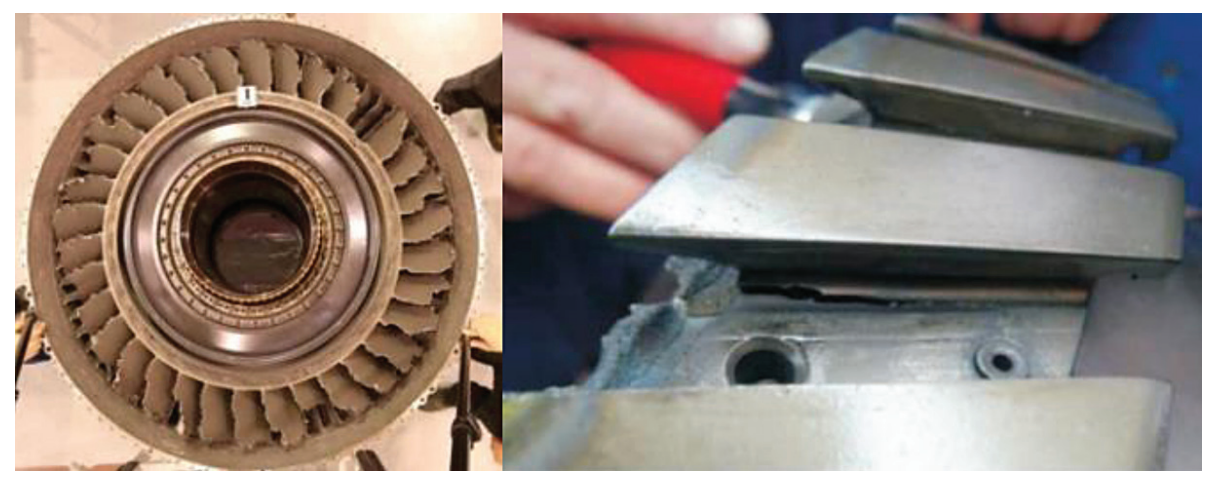

Fig. 1. Examples of fatigue failures of the axial compressor rotor [27, 34]

to be based [24]. Finite elements are then formed by combining the nodes in a predetermined topology (linear, triangular, quadrilateral, tetrahedral or hexahedral) [24]. A more detailed discussion of the theoretical basis of the FEM method can be found in works [2, 20, 21, 24, 28, 30].

In FEM for three-dimensional problems, the displacement vector consists of three components:

$$
u=(u, v, w)
$$

For example, for four-node tetrahedral element, the matrix of nodal displacement values can be presented as follows [3, 18]:

$$
\left\{u^{e}\right\}=\left[u_{1}, u_{2}, u_{3}, u_{4}\right]^{T}
$$

where: $u_{i}=\left(u_{i}, v_{i}, w_{i}\right), i=1,2,3,4$.

The relationship between the strain and displacement, on the other hand, is represented by:

$$
\{\varepsilon\}=\left\{\begin{array}{c}
\varepsilon_{x} \\
\varepsilon_{y} \\
\varepsilon_{z} \\
\gamma_{x y} \\
\gamma_{y z} \\
\gamma_{z x}
\end{array}\right\}=\left\{\begin{array}{c}
\frac{\partial u}{\partial x} \\
\frac{\partial v}{\partial y} \\
\frac{\partial x}{\partial z} \\
\frac{\partial u}{\partial y}+\frac{\partial v}{\partial x} \\
\frac{\partial v}{\partial z}+\frac{\partial w}{\partial y} \\
\frac{\partial w}{\partial x}+\frac{\partial u}{\partial z}
\end{array}\right\}=[T]\{u\}
$$

$[T]$ is commonly referred to as the linear-differential operator [18].

The stress field in the three-dimensional problem is related to the strains as follows:

$$
\{\sigma\}=\left\{\begin{array}{c}
\sigma_{x} \\
\sigma_{y} \\
\sigma_{z} \\
\gamma_{x y} \\
\gamma_{y z} \\
\gamma_{z x}
\end{array}\right\}=[v]\left(\{\varepsilon\}-\left\{\varepsilon_{0}\right\}\right)+\left\{\sigma_{0}\right\}
$$

where: $[v]$ is the elasticity matrix for isotropic materials $[3,18]$.
The basic FEM equation for the element is [3]:

$$
\left[K^{e}\right]\left\{u^{e}\right\}=\left\{Q^{e}\right\}
$$

where: $\left[K^{e}\right]$ is the stiffness matrix $[18,21]$ :

$$
\left[K^{e}\right]=\int_{V}[B]^{T}[v][B] d V
$$

Matrix $[B]$ is called the displacement differentiation matrix. It can be obtained by differentiation of displacements expressed through shape functions and nodal displacements; $V$ is the volume of the element $[18,20]$.

In turn, $\left\{Q^{e}\right\}$ is a load matrix, in which $[N]$ represents shape functions $[3,18]$ :

$$
\left\{Q^{e}\right\}=\int_{A}[N]^{T}\left\{\begin{array}{l}
p_{x} \\
p_{y} \\
p_{z}
\end{array}\right\} d A
$$

For the analyzed model of the dovetail joint, the mesh generated in ANSYS consists of threedimensional ten-node tetrahedral elements, shown in Figure 2.

The created element has ten nodes, each node having three translational degrees of freedom in the nodal $\mathrm{x}, \mathrm{y}$, and z-directions. The displacement field is represented by the following equations [31]: a)
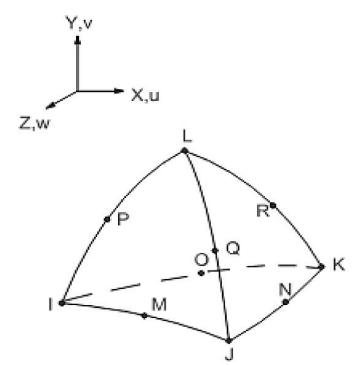

b)

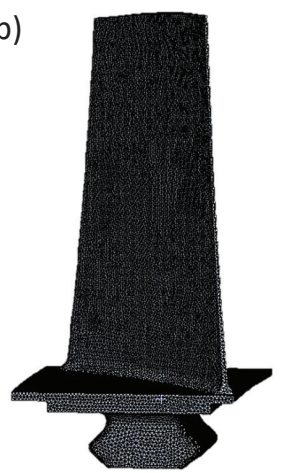

Fig. 2. The ten-node tetrahedral element (a) [31], the blade FE model (b) 


$$
\begin{gathered}
u=u_{I}\left(2 L_{1}-1\right) L_{1}+u_{J}\left(2 L_{2}-1\right) L_{2}+u_{K}\left(2 L_{3}-1\right) L_{3}+u_{L}\left(2 L_{4}-1\right) L_{4}+4 u_{M} L_{1} L_{2} \\
+4 u_{N} L_{2} L_{3}+4 u_{O} L_{1} L_{3}+4 u_{P} L_{1} L_{4}+4 u_{Q} L_{2} L_{4}+4 u_{R} L_{3} L_{4} \\
v=v_{I}\left(2 L_{1}-1\right) L_{1}+v_{J}\left(2 L_{2}-1\right) L_{2}+v_{K}\left(2 L_{3}-1\right) L_{3}+v_{L}\left(2 L_{4}-1\right) L_{4}+4 v_{M} L_{1} L_{2}+4 v_{N} L_{2} L_{3} \\
+4 v_{O} L_{1} L_{3}+4 v_{P} L_{1} L_{4}+4 v_{Q} L_{2} L_{4}+4 v_{R} L_{3} L_{4} \\
w=w_{I}\left(2 L_{1}-1\right) L_{1}+w_{J}\left(2 L_{2}-1\right) L_{2}+w_{K}\left(2 L_{3}-1\right) L_{3}+w_{L}\left(2 L_{4}-1\right) L_{4}+4 w_{M} L_{1} L_{2} \\
+4 w_{N} L_{2} L_{3}+4 w_{O} L_{1} L_{3}+4 w_{P} L_{1} L_{4}+4 w_{Q} L_{2} L_{4}+4 w_{R} L_{3} L_{4}
\end{gathered}
$$

Expressions of the type $N=\left(2 L_{1}-1\right) L_{1}$ are shape functions of corner nodes, while $N=4 L_{3} L_{4}$ are the shape functions of intermediate nodes.

\section{Parameterization of geometry and sensitivity analysis}

The computational model was developed based on the designing requirements to the high pressure compressor of the turbofan engine. The geometry of the elements was captured using the reverse engineering methods. For this purpose, a real blade of the engine and generally available technical documentation were used. The analysis, the geometry of the lock and the rim section of the disc were parameterized. Parameterization of the geometric model and sensitivity analysis made it possible to effectively follow the influence of the variability of the selected parameters on the operation and behavior of a given structure. It enabled developing many variants of the structure, with different geometrical dimensions and features in a relatively short time.
The initial geometry of the lock was characterized by several geometric features (Fig. 3). Taking into account the previous analysis [14] and the condition of dimensional invariance, the following two variables were selected (Fig. 3):

- An angle specifying the width of the lock (the P1 parameter),

- An angle specifying the thickness of the lock (the $\mathrm{P} 2$ parameter).

The analysis allowed determining the influence of the selected features on stress in the lock. It was done using the variability of the operating ranges of the adopted engine, i.e. $60 \%, 85 \%$ and $100 \%$ of the maximum speed of the high pressure rotor $\left(N 2_{\max }=15.183 \mathrm{rpm}\right)$. The equivalent Huber-von Mises-Hencky stress was taken for the analysis. The Von Mises or equivalent stress $\sigma_{e}$ is presented by the following equation $[3,5]$ :

$$
\begin{gathered}
W_{H-M-H}=\frac{1+v}{6 E}\left[\left(\sigma_{1}-\sigma_{2}\right)^{2}+\left(\sigma_{2}-\sigma_{3}\right)^{2}+\left(\sigma_{3}-\sigma_{1}\right)^{2}\right] \\
\sigma_{e}=\left(\frac{1}{2}\left[\left(\sigma_{1}-\sigma_{2}\right)^{2}+\left(\sigma_{2}-\sigma_{3}\right)^{2}+\left(\sigma_{3}-\sigma_{1}\right)^{2}\right]\right)^{\frac{1}{2}}
\end{gathered}
$$

a)

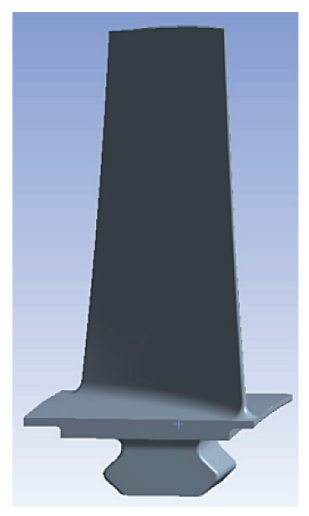

b)

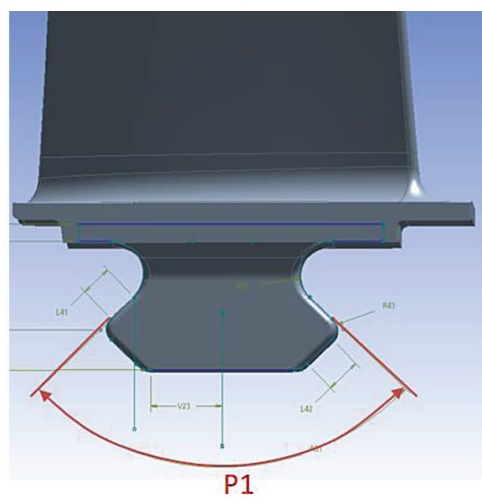

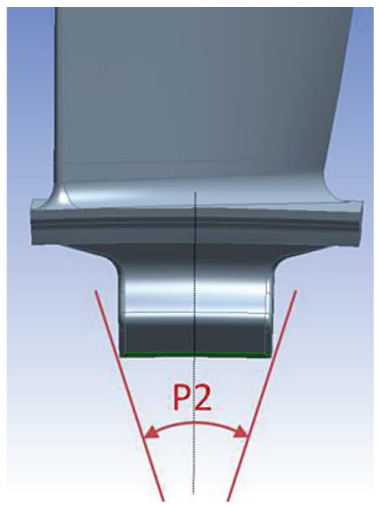

Fig. 3. The geometry of the initial model (a) and the parameters defined (b) 


$$
\sigma_{e}=\left(\frac{1}{2}\left[\left(\sigma_{x}-\sigma_{y}\right)^{2}+\left(\sigma_{y}-\sigma_{z}\right)^{2}+\left(\sigma_{z}-\sigma_{x}\right)^{2}+6\left(\tau_{x y}^{2}+\tau_{y z}^{2}+\tau_{x z}^{2}\right)\right]\right)
$$

The ranges of variability of the considered parameters are presented in Table 1, and the obtained results are shown in Figure 4.

The analysis provided the information on the influence of the P1 and $\mathrm{P} 2$ geometrical parameters on the changes of the equivalent stresses (P4) at different ranges of rotational speed. There is a significant peak of the stress at $\mathrm{P} 1$ values at the range of $90-92^{\circ}$ for engine operation in the idle speed range $60 \% N 2_{\max }$ (Fig. 4a, red). The lowest value of the equivalent stresses occurs at the angle P1 $87-88^{\circ}$. As a result of the increase in the rotational speed (Fig. 4a, green) to the cruising speed range $\left(85 \% N 2_{\max }\right)$, no increase was evidenced. The lowest values of the equivalent stress occur at the range of angle $\mathrm{P} 1$ from $86^{\circ}$ to $88^{\circ}$. For the values above $89^{\circ}$, the values of the stress remain at a similar level; the values are within the range of 750 765 [MPa]. A comparison of these data with results at $85 \% / N 2$ and $100 \% / N 2$ indicated on maximum values of the stress at greater values of $\mathrm{P} 1$ features.

For this parameter, it can be determined that the P1 parameter has very low influence on the equivalent stress values in the joint. In the case of the P2 parameter (Fig. 4b), the region of small

Table 1. Geometric variants of the joint from Figure 3

\begin{tabular}{|c|c|c|c|}
\hline \multirow{2}{*}{ Parameter } & \multirow{2}{*}{ Initial value } & \multicolumn{2}{|c|}{ Range of changes } \\
\cline { 3 - 4 } & & $\min$. & $\max$ \\
\hline $\mathrm{P} 1\left[^{\circ}\right]$ & 92.47 & 86 & 98 \\
\hline $\mathrm{P} 2\left[^{\circ}\right]$ & 1.2 & 1.1 & 1.5 \\
\hline
\end{tabular}

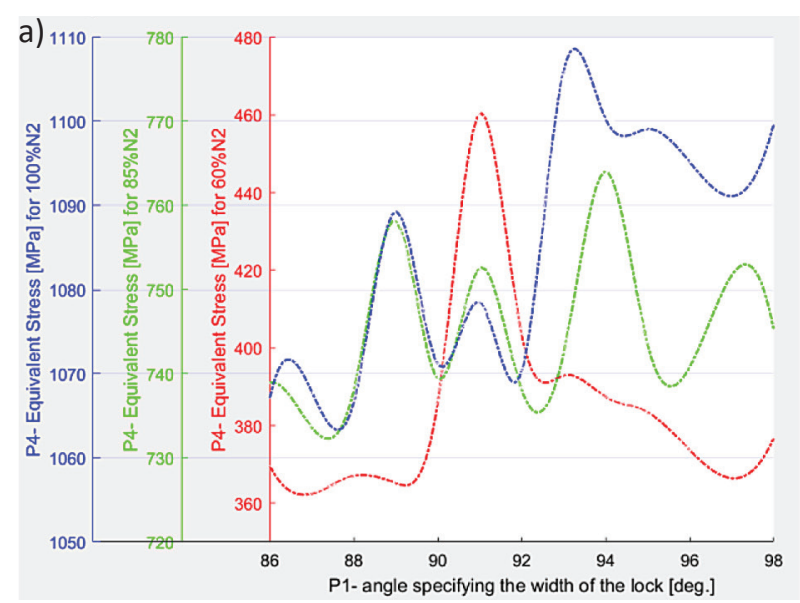

values of the stress was obtained for the $\mathrm{P} 2$ values reaching $1.1-1.35^{\circ}$. A similar course was obtained for all considered ranges of rotational speed. An increase of value of the P2 parameter leads to a gradual increase of the stress in the joint. For this parameter, it can be concluded that the $\mathrm{P} 4$ parameter of the system has a high sensitivity to P2.

\section{PARAMETRIC OPTIMIZATION}

Parametric optimization enables to use the finite element method (FEM) and geometry parameterization to perform optimization of shape, transverse dimensions, material, etc., stress and strain criteria. These methods include non-gradient and gradient algorithms that enable to determine the minimization of single variable functions, as well as the algorithms of minimization in independent or conjugate directions of functions of many variables, etc. [9, 25]. The following analysis used the ANSYS program and the optimization procedures implemented in it. The set of solutions was processed using the Response Surface Optimization tool. As a result of the optimization, two values of the width angle parameter (P1) and the thickness angle of the lock (P2), which for the assumed rotational speed $N 2$ best met the following criteria were distinguished (Table 2):

- The criterion of maximum stress (W1),

- The criterion of minimum strain (W2).

Fig. 4. The influence of geometric parameters P1 (a) and P2 (b) on the equivalent stress at the lock in different ranges of the engine operation 
Table 2. Geometric variants of the blade-disc joint

\begin{tabular}{|c|c|c|}
\hline Parameter & W1 & W2 \\
\hline $\mathrm{P} 1\left[^{\circ}\right]$ & $86(93 \%)$ & $98(106 \%)$ \\
\hline $\mathrm{P} 2\left[^{\circ}\right]$ & $1.5(125 \%)$ & $1.26(105 \%)$ \\
\hline
\end{tabular}

The obtained distributions of the equivalent stress and relative strain of the selected variants are presented in Table 3. The optimization out gave the intended result for both variants. In the case of the initial model, the highest value of the equivalent stress in the joint is close to $490 \mathrm{MPa}$. The maximum value of the stress appearing in the $\mathrm{W} 1$ model is $30 \mathrm{MPa}$ greater than it was calculated for W1. In the case of the W2, $485 \mathrm{MPa}$ represented the maximum value of the stress. In search of a solution for the variant of the minimum strain criterion (W2), the minimum relative strain achieved was at a level of $3.65 \mathrm{e}-04$, while in the case of the base model it was $3.32 \mathrm{e}-04$, and 3.6e-04 for the W1 model. The increase of the value of parameters $\mathrm{P} 1$ and $\mathrm{P} 2$ by only $5-6 \%$ at variant $\mathrm{W} 2$, enabled to significantly reduce the minimum relative strain in the joint considered.

\section{EXPERIMENTAL ANALYSIS}

In order to verify of the results obtained from the numerical analysis, an experiment was carried

Table 3. Distribution of the equivalent stress and strain in the joint

\begin{tabular}{|c|c|c|}
\hline \multicolumn{3}{|c|}{ Rotational speed $85 \% \mathrm{~N} 2 \mathrm{max}$} \\
\hline Sample & Reduced stresses & Relative strains \\
\hline Base model & 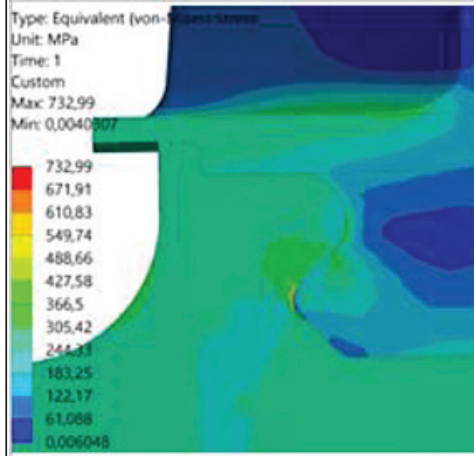 & 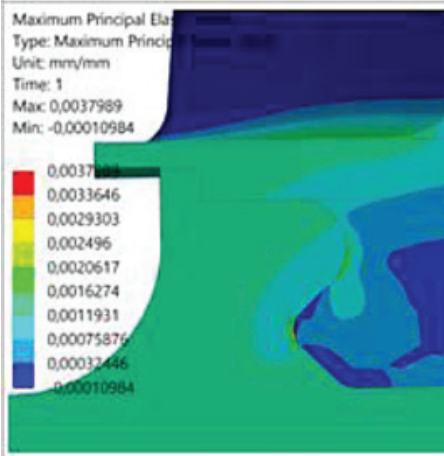 \\
\hline Model W1 & 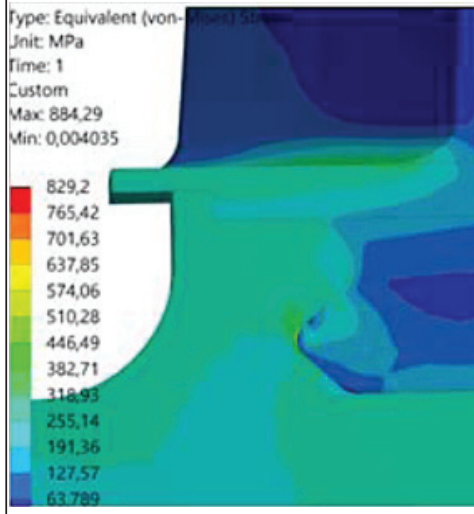 & $\begin{array}{l}\text { Type: Maximum Principe } \\
\text { Unit mm/mm } \\
\text { Time: } 1 \\
\text { Max } 0.0047576 \\
\text { Minc } 0,00018946 \\
0,0047576 \\
0,004207 \\
0,0036582 \\
0,0031086 \\
0,0025589 \\
0,0020092 \\
0,0014596 \\
0,00090989 \\
0.00036017 \\
0,00018946\end{array}$ \\
\hline . & $\begin{array}{l}\text { Type: Equivalent (von-Mises) Stress } \\
\text { Unitim MPa } \\
\text { Time: } 1 \\
\text { Custom } \\
\text { Max } 740,15 \\
\text { Min 0.003844 } \\
730,17 \\
649,04 \\
567,91 \\
496,78 \\
405,65 \\
324,52 \\
243,39 \\
162,26 \\
81,134 \\
0,0048709\end{array}$ & 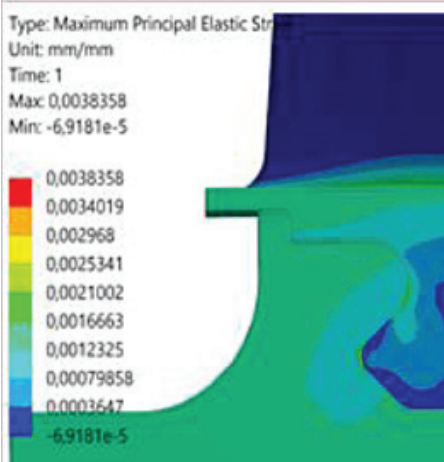 \\
\hline
\end{tabular}



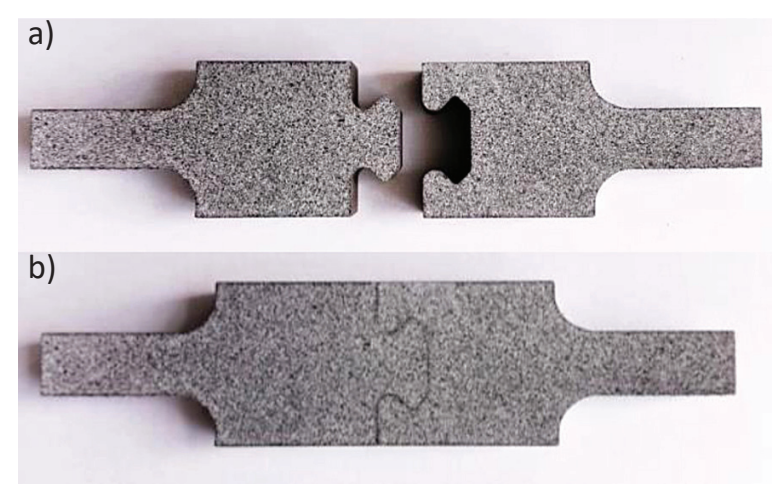

Fig. 5. The lock system before and after connection: (a), (b), respectively

out. It included the production of samples, having geometry of the basic model of the lock and the W1 and W2 variants obtained in the optimization process. The samples were made of low-carbon steel with a density of $7850 \mathrm{~kg} / \mathrm{m}^{3}$. A view of an example of a sample of the lock in the form of a connected and disconnected system is shown in Figure 5. The laboratory tests consisted of a static tensile test. The aim of this experiment was to determine the full fields of strain. The Dantec Q400 digital image correlation system was used to capture distribution of strain. The principal stage of the DIC test was to prepare a special pattern on a measurement zone of a sample, adjust lightening conditions, capture a reference photo of the pattern and follow its variations under loading [6]. In the experiment 60 measurements by means of DIC were done. The loading was used at the range of $0.5-10.0 \mathrm{kN}$. Table 4 shows the selected strain maps. They illustrate the full field strain distribution of total and relative strains, at loading of 5 and $10 \mathrm{kN}$ respectively. The value of $10 \mathrm{kN}$ can be considered as corresponding to the loading of the blade caused by a centrifugal force at the cruising speed for this engine.

The results obtained by means of the DIC system enabled to obtain strain distribution at the following values of tensile force: $5 \mathrm{kN}$ and $10 \mathrm{kN}$. The obtained experimental results are comparable with the results of numerical analysis. The value of stress in the joint of the lock was determined on the basis of the values of relative strains. For example, in the case of the two geometrical variants, at a force of $10 \mathrm{kN}$, the maximum value the stress were between $460-500 \mathrm{MPa}$. It was very close to the stress value determined by means of the numerical approach.

\section{NUMERICAL ANALYSIS}

A characteristic feature of loading in rotor assemblies (including compressor sections) is related to the variations of centrifugal forces caused by changes in the rotational speed. This chapter presents the influence of $\mathrm{P} 1$ and $\mathrm{P} 2$ geometric parameters on the fatigue life of the compressor lock and disc rim, using the geometry parameterization introduced in Chapter 3. Three different speed ranges were considered: $60 \% N 2,85 \% N 2$ and $100 \% N 2$ (N2 - rotational speed of the high

Table 4. Full-field strain maps of the tested samples under loading

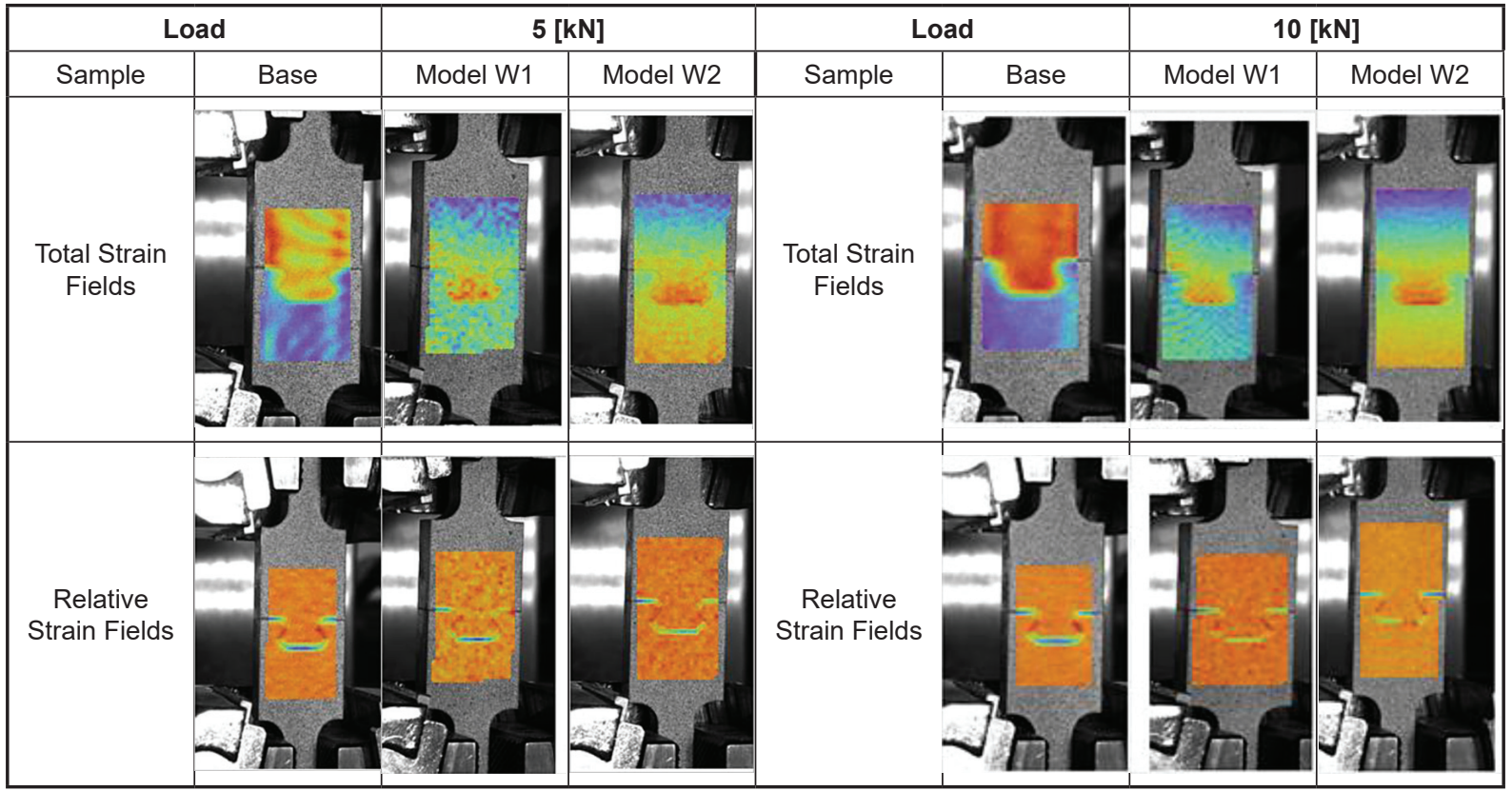


pressure compressor adopted in accordance with the rotational speed range of turbofan engines [26]). Fatigue life of the lock was analyzed for three different engineering materials: typical steel with density $\rho=7850 \mathrm{~kg} / \mathrm{m}^{3}$, the Ti-6Al-4V titanium alloy $\left(\rho=4508 \mathrm{~kg} / \mathrm{m}^{3}\right)$ and Inconel 718 nickel-chromium alloy $\left(\rho=8220 \mathrm{~kg} / \mathrm{m}^{3}\right)$. The mechanical properties of these materials are taken from the study [33]. Because the analysis below only considers the basic load from the tensile force, the simulations were carried out taking zero value of the minimum major stress $\sigma_{\min }$ and the stress coefficient $\mathrm{R}$. In the case of such a load, the mean value of stress $\sigma_{m}$ is equal to the stress amplitude $\sigma_{a}$, so the maximum stress $\sigma_{\max }$ is equal to the doubled value of the stress amplitude $\sigma_{a}$, i.e. $\sigma_{\max }=2 \sigma_{a}[8,13]$. Fatigue strength correction was carried out according to the Goodman's theory. The results of the analysis are included in the charts presented in Figure 6.

Within the considered speed ranges, there were some differences in the influence of the parameters of the increase of fatigue life. In the case of an engine at the RPM $(60 \% N 2)$ and connecting elements made of steel or Inc718 alloy, the reduction in the thickness angle (P2) played a significant role, which gave a clear increase in the durability of the joint.
The influence of parameter P2 on titanium elements occurs at the range of $100 \% \mathrm{~N} 2$, and for values $\mathrm{P} 2>1.35$. It is expressed by a significant decrease of the number of cycles from $10 \mathrm{e} 6$ to $10 \mathrm{e} 5$. For higher rotational speeds $(85 \% N 2$ and $100 \% N 2)$, it can be concluded that the increase in fatigue life is affected by decreasing $\mathrm{P} 2$ at each range of $\mathrm{P} 1$. These results enabled to indicate the range of $\mathrm{P} 2$ value when the super alloy fatigue life decreased significantly, i.e. $1.35 \mathrm{~mm}$. In the range of low and medium engine speeds, the joint made of nickel-chromium alloy are characterized by the longest life. In the range of $60 \% N 2$, this reaches $10 \mathrm{e} 9$ cycles, while at $85 \% N 2$ it is around $10 \mathrm{e} 7$ cycles. It is only at the maximum speed range that the Inconel-based joint is less durable than titanium alloy. However, it should be noticed that the operating time of the engine at the minimum ranges of is smaller than its nominal value because of the thermal conditions.

\section{CONCLUSIONS}

The problem of strength analysis (including the fatigue strength problem) of the lock joint in compressor sections of aircraft turbine engines is a significant problem from the point of safety of flight, which is also present in new constructions.
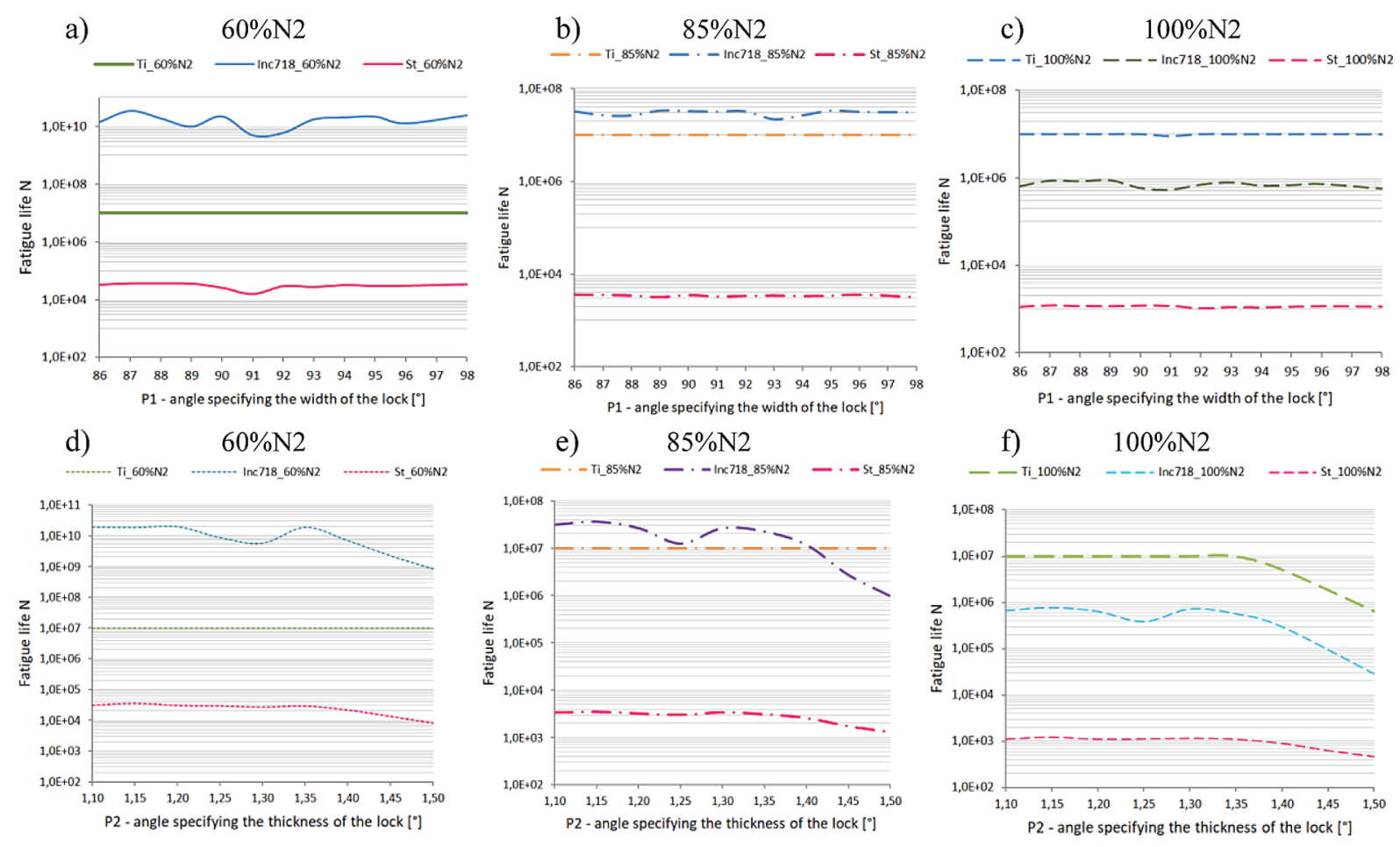

Fig. 6. The influence of the width angle (a, b, c) and the thickness angle (d, e, f) on the fatigue life of the blade-disc joint at three speed ranges and three different materials 
The issue comes down to the attempts to determine the number of elements cycles, subassemblies, assemblies and the complete engine allowing the performance of an aerial task. A detailed description of the significance for the optimization process of individual parameters in the issues related to fatigue life can be obtained by means of the "sensitivity" analysis. A series of analyses were carried out to check the weight of the geometrical parameters of the blade-disc joint defined in point 3 . The studies confirmed that irrespective of the rotational speed adopted, the most important impact (weight) on the fatigue life of the joint was shown by the thickness angle as well as, to a slight degree, the width of the lock. The unequivocal influence of the significance of the lock thickness angle $\mathrm{P} 2$ is observed for higher rotational speeds (the maximum range of engine operation). A very important stage of the research was the validation of the numerical results obtained with the help of an experiment. The physical model and the digital image correlation system were used for this purpose. The results of the conducted experiment confirmed the results of numerical analyses related to optimization. The obtained results of fatigue life should be considered satisfactory; the useful life of the complete CFM56-5 engine, according to the manufacturer's data, is from $0.9 \mathrm{e} 5$ to $3.0 \mathrm{e} 5$ cycles depending on the version [32]. The expected next stages of the work regarding this issue will involve dynamic loads and fatigue failures caused by corrosion or defects resulting from the collisions with sucked-in foreign bodies (FOD).

\section{REFERENCES}

1. Anandavel K., Prakash R. V. Effect of three-dimensional loading on macroscopic fretting aspects of an aero-engine blade-disc dovetail interface. Tribology International, 44, 2011, 1544-1555.

2. Argyris J.H. Energy theorems and structural analysis: A generalized discourse with applications on energy principles of structural analysis including the effects of temperature and non-linear stressstrain relations. Aircraft Engineering and Aerospace Technology, 26(10), 1954, p. 347.

3. Bąk R., Burczyński T., Strength of materials with elements of a computer approach, Warsaw 2009, 378-382. (in Polish)

4. Bednarz A., et al. Influence of initial stress after impact of hard object for fatigue life of the compressor blade. TTS Technika Transportu Szynowego, 12, 2016, 52-55. (in Polish)
5. Bodnar A., Strength of materials. Cracow 2004. (in Polish)

6. Chu T.C., et al. Application of digital-image-correlation techniques to experimental mechanics. Experimental Mechanics, 25(3), 1985, 232-244.

7. Dębski M., Dębski D., Selected issues of fatigue strength of aircraft structures, Warsaw 2014, 318325. (in Polish)

8. Gasiak G., Pawliczek R. Fatigue strength of a structural steel under asymmetric loading. Zeszyty Naukowe. Mechanika / Politechnika Opolska, 2002, 25-41. (in Polish)

9. Kachel S. The method of multi-criteria design of the airplane body including the mission. Warsaw 2011. (in Polish)

10. Kermanpur A., et al. Failure characterization of Ti6Al4V gas turbine compressor blades. WIT Transactions on Engineering Sciences, 57, 2007, 383-392.

11. Kheto M.K., et al. Fretting fatigue analysis in dovetail joint of compressor through numerical simulation. SASTECH, 8, 2009, 71-76.

12. Kłysz S. Fatigue life analysis of construction elements used in aviation. Technical Sciences, 8, 2005, 181-192.

13. Kocańda S., Szala J. Basics of fatigue calculations, Warsaw 1997. (in Polish)

14. Kozakiewicz A., Grzejszczak O. Influence of selected geometric parameters on strength properties of compressor's jet engine blade joint. Mechanik 07, 2016, 740-741. (in Polish)

15. Lourenco N.J., Graca M.L.A, et al., Fatigue failure of a compressor blade, Engineering Failure Analysis 15, 2008, 1150-1154.

16. Łagosz M., Szczeciński S. Construction of aircraft engines. Selected issues of strength and dynamics of the structure. Warsaw 1985. (in Polish)

17. Merquid S. A., et al. Theoretical and experimental studies of structural integrity of dovetail joints in aeroengine discs. Journal of Materials Processing Technology, 56, 1996, 668-677.

18. Moaveni S., Finite element analysis. Theory and application with ANSYS, New Jersey 1999, 440-447.

19. Nakhodchi S., et al. Fatigue life prediction in the damaged and un-damaged compressor blades. Engineering Solid Mechanics, 2013, 43-50.

20. Nikishkov G.P., Introduction to the finite element method. Japan 2004, 16-17.

21. Nowotarski I., Static and dynamic calculations of aircraft turbine engines by finite element method, Warsaw 2001.

22. Papanikos P. Three-dimensional nonlinear finite element analysis of dovetail joints in aeroengine discs. Finite Elements in Analysis and Design, 29, 1998, 173-186. 
23. Prevéy P.S., Jayaraman N. et al. Mitigation of fretting fatigue damage in blade and disk pressure faces with low plasticity burnishing, ASME Lambda Technologies, USA Ohio 2007.

24. Saxena A., Sahay B. Computer aided engineering design. Springer 2005, 309-310.

25. Stadnicki J. Theory and practice of solving optimization problems with examples of technical applications. Warsaw 2006. (in Polish)

26. Szczeciński S., et al. Aircraft turbine engines, construction - operation - diagnostics. Warsaw 2010. (in Polish)

27. Trelka, M., et al. Selected problems of RD-33 engine reliability in operation. Combustion engines, 165(2), 2017, 33-40.
28. Turner M.J. Clough R.C., Martin H.C., Topp L.J. Stiffness and deflection analysis. Journal of Aero Sciences, 23, 1956, 805-823.

29 . Witoś M. Increasing the durability of turbine engines through active diagnostics and control. Warsaw 2010. (in Polish)

30. Zienkiewicz O.C. The finite element method in engineering science. Warsaw, 1972.

31. ANSYS v.18 documentation.

32. CFM Flight Ops Support B737. Conference Flight Operations Support. 2005.

33. Metallic materials and elements for aerospace vehicle structures, MIL-HDBK-5H, 1998.

34. www.avherald.com 